\title{
COMPACT COMPATIBLE TOPOLOGIES FOR GRAPHS WITH SMALL CYCLES
}

\author{
VICTOR NEUMANN-LARA AND RICHARD G. WILSON
}

Received 11 November 2004 and in revised form 12 July 2005

A topology $\tau$ on the vertices of a comparability graph $G$ is said to be compatible with $G$ if each subgraph $H$ of $G$ is graph-connected if and only if it is a connected subspace of $(G, \tau)$. In two previous papers we considered the problem of finding compatible topologies for a given comparability graph and we proved that the nonexistence of infinite paths was a necessary and sufficient condition for the existence of a compact compatible topology on a tree (that is to say, a connected graph without cycles) and we asked whether this condition characterized the existence of a compact compatible topology on a comparability graph in which all cycles are of length at most $n$. Here we prove an extension of the above-mentioned theorem to graphs whose cycles are all of length at most five and we show that this is the best possible result by exhibiting a comparability graph in which all cycles are of length 6 , with no infinite paths, but which has no compact compatible topology.

\section{Introduction and preliminary results}

Recall that a graph is a comparability graph if and only if it is the graph of some poset. More formally if we define the graph of a poset $(X,<)$, denoted by $G((X,<))$, on the vertex set $X$, by

$$
[x, y] \in E(\mathscr{G}((X,<))) \text { if and only if either } x<y \text { or } y<x
$$

then $G$ is a comparability graph if it is isomorphic to $\mathscr{G}((X,<))$ for some poset $(X,<)$. Note that $G$ is a functor from the category of posets onto the category of comparability graphs with reflexive graph-homomorphisms as morphisms. Thus if $G$ is a comparability graph, then there is some (nonunique) poset $(X,<)$ such that $\mathscr{G}(X,<)=G$ (thus $X=$ $V(G)$ ) and so $\mathscr{G}$ induces orderings on the vertices of $G$ (or equivalently, orientations of the edges of $G$ ). In the sequel, a comparability graph $G$ with a (fixed) induced order < will be denoted by $(G,<)$ (even though strictly speaking, the order is on the set $V(G)$ ) and we refer to such a graph as an ordered comparability graph; clearly the category of posets and the category of ordered comparability graphs are isomorphic. 
Préa introduced the notion of compatibility between a graph $G$ and a topology on its vertex set $V(G)$ in [7]. There, a topology $\tau$ on $V(G)$ was said to be compatible (resp., weakly compatible) with a graph $G$ if every induced subgraph $H$ of $G$ (resp., every finite induced subgraph $H$ of $G$ ) is connected if and only if the relative topology induced by $\tau$ on $V(H)$ is connected. It was shown that, in the case of a locally finite comparability graph $G$ with a finite number of components, a compatible topology exists if and only if $G$ is a comparability graph. This result was generalized to arbitrary comparability graphs in [5]. Clearly, if a topology $\tau$ on the vertex set $V(G)$ of a graph $G$ is weakly compatible, then the graph structure of $G$ is determined by the topology in the sense that whenever $x, y$ are distinct vertices of $G,[x, y] \in E(G)$ (the edge set of $G$ ) if and only if $x \in \operatorname{cl}_{\tau}\{y\}$ or $y \in \operatorname{cl}_{\tau}\{x\}$.

We will be studying topologies on the vertex sets of ordered comparability graphs and in order to relate the topology to the partial order, we require that the specialization order of the topology $\tau$ (which we denote by $\ll_{\tau}$ and is defined by $x \ll_{\tau} y$ if and only if $x \in \mathrm{cl}_{\tau}\{y\}$ ) exactly coincide with the given partial order. Such a topology will be said to be consistent with the poset or ordered comparability graph. Clearly a consistent topology must be weakly compatible in the sense of $[5,7]$, but not vice versa. In what follows, in order to avoid unnecessary terminology, we assume that the conditions of weak compatibility and compatibility include the condition of consistency. Thus we make the following formal definitions.

A topology $\tau$ on the vertex set of an ordered comparability graph $(G,<)$ is said to be compatible (resp., weakly compatible) if for each induced subgraph $H$ of $G$ (resp., each finite induced subgraph $H$ of $G$ ), $H$ is $\tau$-connected if and only if it is graph-connected and $\ll_{\tau}$ coincides with $<$.

Recall from [6] that a graph $G$ is said to be cyclically bounded if there is some $k \in \mathbb{N}$ (the set of positive integers) such that every cycle in $G$ is of length at most $k$. In [6, Theorem 3.1], we have shown that an (ordered) tree possesses a compact compatible topology if and only if it has no infinite paths and we asked whether a similar theorem holds in the case of cyclically bounded ordered comparability graphs.

If $\leq$ is a partial order on a set $X$, then we define $(\leftarrow, x]=\{y: y \leq x\}$ and $[x, \rightarrow)=\{y$ : $y \geq x\}$; the weak topology $\omega$ on $X$ is the topology generated by the sets $\{X \backslash(\leftarrow, x]: x \in X\}$ and the Alexandroff topology $\alpha$ induced by the partial order is that topology with base $\{[x, \rightarrow): x \in X\}$. It was shown in [5, Theorem 1.10] that if $\mu$ is a compatible topology for a poset $(X,<)$, then $\omega \subseteq \mu \subseteq \alpha$.

For $w \in V(G), N(w)$ will denote the set of graph neighbours of $w$ and a graph $G$ is said to be blunt if for each $v \in V(G), G \backslash N(v)$ has only a finite number of components. Finally, say that a set of vertices $H$ of a graph $G$ is centred if there exists $v \in G$ such that $H \subseteq N(v) \backslash\{v\} . v$ is then said to be a centre for $H$. Say that $H$ is finitely centred if every finite subset of $H$ is centred.

For all other undefined topological and graph-theoretical terms we refer the reader to $[1,2,3]$.

THEOREM 1.1. If an ordered bipartite graph $(G,<)$ either

(1) has no infinite paths, or

(2) is cyclically bounded,

then the weak topology is compact. 
Proof. We denote the set of minimal (resp., maximal) elements of the order by $\min (G)$ (resp., $\max (G)$ ). Suppose to the contrary that the weak topology is not compact; then by [6, Theorem 2.12], there is some (necessarily infinite) subset $V \subseteq \max (G)$ of maximal elements of $(G,<)$ which is finitely centred but not centred. Choose $v_{0} \in V$ and $m_{0} \in \min (G)$ such that $m_{0}<v_{0}$. Suppose now that we have chosen distinct vertices $v_{0}, v_{1}, \ldots, v_{n} \in V$ and $m_{0}, \ldots, m_{n} \in \min (G)$ such that

(a) $m_{k}$ is a centre for $\left\{v_{0}, v_{1}, \ldots, v_{k}\right\}$ for each $k \in\{0,1, \ldots, n\}$, and

(b) $\left[m_{k}, v_{k+1}\right] \notin E(G)$ for each $k \in\{0,1, \ldots, n-1\}$,

then clearly

$$
T=m_{0}, v_{0}, m_{1}, v_{1}, \ldots, m_{n}, v_{n}
$$

is a path in $G$ of length $2 n+1$ and

$$
v_{0}, m_{1}, v_{1}, \ldots, v_{n-1}, m_{n}
$$

is a cycle of length $2 n$. We extend this path to a path of length $2 n+3$ thus showing by induction that there is an infinite path in $G$ and at the same time construct a cycle of length $2 n+2$, showing that $(G,<)$ is not cyclically bounded.

Since $m_{n}$ is not a centre for $V$, there is some $v_{n+1} \in V$ such that $m_{n} \npreceq v_{n+1}$ and then necessarily $v_{n+1} \notin\left\{v_{0}, v_{1}, \ldots, v_{n}\right\}$ by (a) above. However, $V$ is finitely centred and hence there is some $m_{n+1} \in \min (G)$ such that $m_{n+1}<v_{k}$ for all $k \in\{0,1, \ldots, n, n+1\}$; it follows from (b) that $m_{n+1} \notin\left\{m_{0}, m_{1}, \ldots, m_{n}\right\}$ and hence we have extended the path $T$ to a path of length $2 n+3, m_{0}, v_{0}, m_{1}, v_{1}, \ldots, m_{n}, v_{n}, m_{n+1}, v_{n+1}$. Note also, that since $\left[m_{n+1}, v_{0}\right] \in E(G)$, we have also constructed a cycle

$$
v_{0}, m_{1}, v_{1}, \ldots, v_{n-1}, m_{n}, v_{n}, m_{n+1}
$$

of length $2 n+2$.

We recall the following definitions from [6].

A poset (or equivalently, an ordered comparability graph) $(X,<)$ will be said to be basal if the set of minimal elements of the order, $\min (X)$, is coinitial. Let $(X,<)$ be a basal poset; we define a new order $\prec$ on $X$ as follows:

$$
x \prec y \quad \text { if and only if } x<y \text { and } x \in \min (X) \text {. }
$$

The poset $(X, \prec)$ will be called the bipartite skeleton of $(X,<)$. Similarly, $\mathscr{G}(X, \prec)$ will be called the ordered bipartite skeleton of $\mathscr{G}(X,<)$; it is easy to see that $\mathscr{G}(X, \prec)$ is an (ordered) bipartite graph.

In $[6$, Section 2], we showed that the existence of a compact compatible (or weakly compatible) topology on such a graph is equivalent to the existence of such a topology on its bipartite skeleton [6, Theorems 2.14 and 2.18].

Corollary 1.2. If an ordered comparability graph has no infinite paths or is cyclically bounded, then it has a compact weakly compatible topology. 
Proof. Suppose $(G,<)$ is an ordered comparability graph which is either cyclically bounded or has no infinite paths; we claim that $G$ is basal. For if not, then there is some descending chain $x_{0}>x_{1}>x_{2}>\cdots$ of vertices which implies the existence both of an infinite path and arbitrarily large cycles in $G$. Thus $(G,<)$ has a bipartite skeleton which we denote by $(H, \prec)$. Since $H$ is a subgraph of $G$, it also is either cyclically bounded or has no infinite paths and hence by the theorem, the weak topology on $(H, \prec)$ is compact. The result now follows from [6, Theorem 2.14].

COROLlary 1.3. The weak topology corresponding to either of the two possible orderings of a tree is compact.

Let us say that a bipartite graph is (weakly) bilaterally compact if for each of the two possible orderings there is at least one compact (weakly) compatible topology.

The following result is now clear.

Corollary 1.4. A bipartite graph which either has no infinite paths or is cyclically bounded is weakly bilaterally compact.

Thus a bipartite graph which is not weakly bilaterally compact must have both an infinite path and arbitrarily large cycles_ _ such a graph is given as [6, Example 2.19]. Notice that for this graph neither of the two weak topologies corresponding to the two possible orderings of the graph are compact. However, by modifying slightly the above-mentioned example, we obtain a bipartite graph which has a compact compatible topology with respect to one ordering, but which does not even have a weakly compatible compact topology with respect to the other.

Example 1.5. The graph $G$ is defined by

$$
\begin{gathered}
V(G)=(\mathbb{N} \times\{0\}) \cup((\mathbb{N} \cup\{\infty\}) \times\{1\}), \\
E(G)=\{[(m, 0),(n, 1)]: m, n \in \mathbb{N}, m \neq n\} \cup\{[(\infty, 1),(n, 0)]: n \in \mathbb{N}\} .
\end{gathered}
$$

It is easy to see that with the ordering in which vertices whose second coordinate is 0 are minimal, the weak topology restricted to the set of minimal elements $\{(n, 0): n \in \mathbb{N}\}$ is discrete (exactly as in [6, Example 2.19]) and hence the weak topology is not compact. However, with the reverse order, the vertex $(\infty, 1)$ is a centre for the set of maximal elements $\{(n, 0): n \in \mathbb{N}\}$ and hence by [6, Corollary 2.10], for this ordering there is a compatible compact topology on $G$.

\section{Graphs whose cycles are small}

We have shown in $[6$, Theorem 3.4$]$ that if $(G,<)$ is a cyclically bounded ordered comparability graph for which there exists a compact compatible topology, then $G$ has no infinite paths. Hence we were led to ask the following question, the answer to which will be given in this section.

Problem 2.1. If a cyclically bounded ordered comparability graph has no infinite paths, then does it have a compatible compact topology? 
Recall that a block in a graph $G$ is a maximal 2-connected induced subgraph; a block is trivial if it contains only two vertices (and hence one edge). A well-known construction (see [3, page 36]) is the block-cut-point tree whose vertices are the set of blocks together with the set of cut-points of $G$ and whose edges correspond to the adjacency of blocks and cut-points in the original graph. In the sequel, $K_{\alpha, \beta}$ denotes the complete bipartite graph on $\alpha$ and $\beta$ vertices.

Theorem 2.2. Let $G$ be a 2-connected ordered bipartite graph in which all cycles are of length at most 4; then $G$ is bilaterally compact.

Proof. If $G$ is a trivial block, then the result is clear. If not, then we claim that $G=K_{2, \gamma}$ for some cardinal $\gamma>1$. To see this, consider the sets $\min (G)$ and $\max (G)$ of minimal and maximal elements of $G$, respectively. Since each pair of points in a 2-connected graph lie in a cycle, whenever $v \in \min (G)$ and $w \in \max (G)$, there is some 4-cycle containing $v$ and $w$ and hence $[v, w] \in E(G)$; thus $G$ is a complete bipartite graph. However, if both $\min (G)$ and $\max (G)$ have at least three elements, then $G$ contains a copy of the complete bipartite graph $K_{3,3}$ and hence contains a cycle of length 6 , thus proving our claim. To complete the proof of the theorem, it remains only to show that for each cardinal $\gamma$, the graph $K_{2, \gamma}$ is bilaterally compact; that is to say, for either of the two possible orderings of $K_{2, \gamma}$, there is a compact compatible topology on $K_{2, \gamma}$. However, if the set of minimal elements is finite, then the Alexandroff topology corresponding to that ordering is a compact compatible topology. If on the other hand, the set of minimal elements is infinite, then letting $\alpha$ denote the Alexandroff topology induced by the ordering, we can define a topology $\sigma$ on $G=K_{2, \gamma}$ as follows: fix $v_{0} \in \min (G)$, then

$$
U \in \sigma \quad \text { if and only if } U \in \alpha \text { and if } v_{0} \in U \text {, then } G \backslash U \text { is finite. }
$$

If $\gamma$ is infinite, then the relative topology which $\sigma$ induces on $\min (G)$ is homeomorphic to the one-point compactification of the discrete space of cardinality $\gamma$ and hence $\sigma$ is compact. We leave the routine verification that $\sigma$ is compatible to the reader.

Remark 2.3. We note for future reference that the topology $\sigma$ constructed in the previous theorem on the 2-connected ordered bipartite graph $G=K_{2, \gamma}$ has (at least) one minimal vertex all of whose neighbourhoods are cofinite. If the set of <-minimal elements of $G$ is finite, then the topology on $G$ is precisely the Alexandroff topology $\alpha$ induced by the order <; if on the other hand, the set of minimal elements is infinite, then all vertices but one (the vertex $v_{0}$ of Theorem 2.2) have Alexandroff neighbourhoods. In either case, $\sigma$ will be termed a canonical topology for the block $G$. A minimal vertex all of whose neighbourhoods are cofinite in $G$ will be called determining for $G$. Hence, if $\sigma$ is a canonical topology for $G$, then either there are a finite number of minimal vertices, all of which are determining or there is a unique minimal vertex which is determining, in which case this vertex will be termed special. It is also clear that every maximal element of $G$ is $\sigma$-open and each minimal element is $\sigma$-closed.

The following lemma shows that in the search for compact compatible topologies, we may restrict our attention to connected graphs. 
LEMMA 2.4. If each graph component of an ordered comparability graph $(G,<)$ has a compact compatible topology, then so does $(G,<)$.

Proof. Let $\left\{C_{\alpha}: \alpha \in I\right\}$ be the set of graph components of $(G,<)$ and let $\sigma_{\alpha}$ be a compact compatible topology for $C_{\alpha}$. If $I$ is finite, then the disjoint topological union of the spaces $\left(C_{\alpha}, \sigma_{\alpha}\right)$ is the required compact space. If on the other hand $I$ is infinite, then fix $\alpha \in I$ and $x \in C_{\alpha}$ and define a topology $\tau$ on $G$ as follows:

$$
\begin{aligned}
& U \in \tau \quad \text { if and only if } U \cap C_{\alpha} \in \sigma_{\alpha} \text { and if } x \in U, \\
& \text { then } U \supseteq \bigcup\left\{C_{\alpha}: \alpha \in I \backslash F\right\} \text { for some finite } F \subseteq I .
\end{aligned}
$$

We omit the straightforward proof that $\tau$ is a compact compatible topology for $(G,<)$.

In order to prove our main theorem, we need some more notation. Suppose that $G$ has no infinite paths, then $G$ has a non-cut-point. Let $B_{0}$-the root block of $G$-be the block containing the non-cut-point which we denote by $v_{B_{0}}$. Every block $C$ of $G$ distinct from $B_{0}$ contains a unique cut-point $v_{C}$ of $G$ whose distance from $v_{B_{0}}$ is minimal among all other vertices of $C$. Say that a block $C$ is an immediate successor block to a block $B$ (or equivalently that $B$ is an immediate predecessor to $C$ ) if $B \cap C \neq \varnothing, v_{B} \neq v_{C}$, and every path from $v_{C}$ to $v_{B_{0}}$ contains $v_{B}$. Each block other than $B_{0}$ has a unique immediate predecessor, but in general may have many immediate successors. Finally, say that a block $C$ is a successor to a block $B$ if there is a finite sequence of blocks $B=B_{0}, B_{1}, \ldots, B_{n}=C$ such that $B_{k+1}$ is an immediate successor of $B_{k}$ for each $k \in\{0,1, \ldots, n-1\}$.

Theorem 2.5. Let $(G,<)$ be a connected ordered bipartite graph in which all cycles are of length no greater than 4 . Then $(G,<)$ has a compact compatible topology if and only if the associated block-cut-point tree has no infinite paths.

Proof. Suppose that $(G,<)$ is such a connected ordered bipartite graph whose associated block-cut-point tree has no infinite paths.

Using the notation of the paragraph preceding this theorem, the ordering < on $G$ determines an ordering $<_{B_{0}}$ on the root block $B_{0}$. Let $\sigma_{0}$ be a canonical compact topology for $B_{0}$ in which, if the non-cut-point $v_{B_{0}}$ (henceforth denoted by $v_{0}$ ) is minimal in $(G,<)$, then it is a determining vertex for $\left(B_{0}, \sigma_{0}\right)$. If $G$ has no cut-points, then $G$ consists of the single bilaterally compact block $B_{0}$ and we define $\tau=\sigma_{0}$ and we are done. Otherwise, we proceed to define a topology $\sigma$ on $G$ as follows.

Let $\left\{B_{\alpha}: \alpha \in \kappa\right\}$ be a well-ordering of the blocks of $G$ in such a way that $B_{0}$ is the root block fixed previously and for $\alpha>0$, let $v_{\alpha}$ be the cut-point of $G$ in $B_{\alpha}$ whose distance from $v_{0}$ is minimal.

The ordering < on $G$ determines an ordering $<_{B}$ on each block $B$ of $G$. Since each block $B$ is either trivial or isomorphic to $K_{2, \gamma}$ for some cardinal $\gamma$ and each block has a unique immediate predecessor, it is clear that we can choose a canonical compact compatible topology $\sigma_{\alpha}$ on $B_{\alpha}$ (in which every maximal element of $\left(B_{\alpha},<_{B_{\alpha}}\right)$ is $\sigma_{\alpha}$-open and each minimal element is $\sigma_{\alpha}$-closed) and such that 
(1) if $v_{\alpha}$ is a minimal vertex of the block $B_{\alpha}$, then $\sigma_{\alpha}$ is chosen in such a way that $v_{\alpha}$ is a determining vertex for $B_{\alpha}$ (and thus if $B_{\alpha}$ has an infinite number of minimal vertices, $v_{\alpha}$ will be the unique special vertex of $\left.\left(B_{\alpha}, \sigma_{\alpha}\right)\right)$.

We now define a topology $\sigma$ on $G$ by

$$
U \in \sigma \quad \text { if and only if } U \cap B_{\alpha} \in \sigma_{\alpha} \text { for each block } B_{\alpha} \text { of } G \text {. }
$$

A moment's reflection now shows that the topology $\sigma$ is well defined and that

(2) each vertex which is maximal with respect to $<$ is $\sigma$-open, and each vertex which is minimal with respect to $<$ is $\sigma$-closed; furthermore, for each $\alpha \in \kappa, \sigma \mid B_{\alpha}=\sigma_{\alpha}$;

(3) if $z$ is minimal (resp., maximal), then the graph components of $G \backslash\{z\}$ are $\sigma$ open (resp., $\sigma$-closed) in $G$. Furthermore, for each $z \in G$, the graph components of $G \backslash\{z\}$ are open and closed in $G \backslash\{z\}$;

(4) if $z$ is a maximal (resp., minimal) vertex of $G$, then $o(z)=\cup\left\{B_{\beta}\right.$ : every path from $v_{\beta}$ to $v_{0}$ contains $z$ \} is $\sigma$-open (resp., $\sigma$-closed). $(o(z)$ may be thought of as those points which are separated from $v_{0}$ by $z$ together with $z$ itself.)

The topology $\sigma$ on $G$ is weakly compatible since if $[v, w] \in E(G)$, it follows that $v, w \in$ $B_{\alpha}$ for some block $B_{\alpha}$ of $G$ and then by (2), since $\sigma_{\alpha}$ is a compatible topology for $B_{\alpha}$, it follows that $\{v, w\}$ is $\sigma_{\alpha}$-connected and hence $\sigma$-connected. Conversely, if $[v, w] \notin E(G)$, then there are two possibilities. If $v, w$ lie in some block $B_{\alpha}$ of $G$, then since $\sigma_{\alpha}$ is a compatible topology for $B_{\alpha}$, it follows again from (2) that $\{v, w\}$ is not $\sigma$-connected. If there is no block of $G$ containing both $v$ and $w$, then there is some cut-point $z$ of $G$ such that $v$ and $w$ lie in different graph components of $G \backslash\{z\}$. Condition (3) now shows that the relative $\sigma$ topology on $\{v, w\}$ is discrete.

We claim that $\sigma$ is a compatible topology for $G$. To show this, by [6, Lemma 2.7], it suffices to show that if $H$ is a $\sigma$-connected subset of $G$ and $v$ is a vertex of $G$ such that $N(v) \cap H=\varnothing$, then $v \notin \mathrm{cl}_{\sigma}(H)$. Now, to prove our claim, note that by Remark 2.3, if $v \in G$ is not a special determining vertex of any block, then each $\sigma$-neighbourhood of $v$ in $G$ is an Alexandroff neighbourhood of $v$ in $G$ and hence $v$ has a neighborhood contained in $N(v)$. Hence if we assume that $N(v) \cap H=\varnothing$ and $v \in \mathrm{cl}_{\sigma}(H)$, then $v$ must be a special vertex of some block. However, if $v$ is special in some block, then each $\sigma$-neighborhood of $v$ contains all maximal vertices and all but finitely many minimal vertices of that block and so $H$ must meet $o(z)$ for infinitely many minimal (nonspecial) vertices $z(\neq v)$ lying in some block $B$ in which $v$ is special. By (4), each such $o(z)$ is $\sigma$-closed and $o(z) \backslash\{z\}$ is $\sigma$ open; since $H$ cannot contain any maximal vertices of $B$ (they all lie in $N(v)$ ), $H \cap o(z)=$ $H \cap(o(z) \cup N(z))$ is a proper open and closed subset of $H$, a contradiction.

We now wish to modify the topology $\sigma$ so as to obtain a compact compatible topology $\tau$ on $G$. Let $w_{0}$ be a determining vertex for $B_{0}$ and for each $\alpha>0$ pick a determining vertex in $B_{\alpha}$, with the condition that if $v_{\alpha}$ is closed (and hence by the definition of the topology $\sigma_{\alpha}, v_{\alpha}$ is determining in $B_{\alpha}$ ), then $v_{\alpha}$ is the determining vertex so chosen. Denote by $W$ this set of determining vertices and for each distinct vertex $w \in W$, we fix a block $B_{\alpha}$ such that $w$ is determining in $B_{\alpha}$. There may be many such blocks, but we assume that if $w$ is special in some block, then $B_{\alpha}$ is chosen so that $w$ is special in $B_{\alpha}$ and also that the distance from $v_{0}$ to $v_{\alpha}$ is minimal among all blocks in which $w$ is special; in other words, if $w$ is special in some block, then $B_{\alpha}$ is chosen so that $w$ is special in $B_{\alpha}$ but not in the 
immediate predecessor block of $B_{\alpha}$. Also, if $z$ is a vertex of $G$, then we denote the set of graph components of $G \backslash\{z\}$ which do not contain $v_{0}$ by $\mathscr{C}_{z}$; it follows from (3) that if $z$ is maximal (resp., minimal), then each element of $\mathscr{C}_{z}$ is closed (resp., open) in $(G, \sigma)$.

Now for each $w \in W$ and each block $B_{\alpha}$ so chosen and for each finite set $F$ of minimal vertices in $o\left(v_{\alpha}\right) \backslash\{w\}$, each finite set $K$ of maximal vertices of $o\left(v_{\alpha}\right) \backslash\{w\}$ and each finite set $\mathscr{H} \subseteq \cup\left\{\mathscr{b}_{z}: z \in K\right\}$, we define

$$
V(w, F, K, \mathscr{H})=\left(N(w) \cup o\left(v_{\alpha}\right)\right) \backslash \cup\{o(z): z \in F\} \backslash \cup \mathcal{H} .
$$

If $w \neq v_{\alpha}$, then $v_{\alpha}$ is maximal and hence by (4), $o\left(v_{\alpha}\right)$ is $\sigma$-open and $N(w) \subseteq o\left(v_{\alpha}\right)$. While if $w=v_{\alpha}$, then since $w$ is not special in the immediate predecessor block of $B_{\alpha}$, it follows again that $N(w) \cup o(w)$ is a $\sigma$-open neighbourhood of $w$. Thus in both cases, it follows from (3) and (4) that for each vertex $w \in W, V(w, F, K, \mathcal{H})$ is $\sigma$-open. We now define

$$
\begin{aligned}
& \mathscr{B}=\{U \in \sigma: \text { whenever } w \in W \cap U \text {, then } U \supseteq V(w, F, K, \mathscr{H}) \\
& \text { for some finite set of minimal vertices } F \subseteq o\left(v_{\alpha}\right) \backslash\{w\}, \\
& \text { some finite set of maximal vertices } K \subseteq o\left(v_{\alpha}\right) \backslash\{w\} \\
& \text { and some finite set } \left.\mathscr{H} \subseteq \cup\left\{\mathscr{C}_{z}: z \in K\right\}\right\} .
\end{aligned}
$$

After a moment's thought it is clear that $\mathscr{B}$ is closed under finite intersections and hence $\mathscr{B}$ is a base for a topology which we denote by $\tau$. Note also that if $B_{\alpha}$ is a block of $G$ and $w \in B_{\alpha}$ is determining in $B_{\alpha}$, then $w$ has a $\sigma$-open neighbourhood $U$ such neighbourhood $U$ such that $B_{\alpha} \backslash U$ consists of minimal vertices. Moreover, every other point of $B_{\alpha}$ has a $\sigma$-neighborhood which misses $w$ and hence $\tau\left|B_{\alpha}=\sigma\right| B_{\alpha}$ and clearly $\tau \subseteq \sigma$.

Finally it is clear from the definition of $\tau$ that

(5) if $z$ is a maximal vertex, then each element $C \in \mathscr{C}_{z}$ is $\tau$-closed and $C \cup\{z\}$ (and hence $o(z))$ is $\tau$-open, and if $z$ is a minimal vertex, then each element $C \in \mathscr{C}_{z}$ is $\tau$-open and $o(z)$ is closed.

We will prove that $\tau$ is a compact compatible topology for $(G,<)$. To this end, we show first that $\tau$ is a weakly compatible topology for $G$. However, if $[x, y] \in E(G)$, then there is some block $B_{\alpha}$ of $G$ such that $x, y \in B_{\alpha}$ and hence $\{x, y\}$ is $\sigma_{\alpha}$-connected and hence $\tau$-connected. Conversely, if $[x, y] \notin E(G)$ and $x, y$ lie in some block $B_{\alpha}$ of $G$, then since $\sigma_{\alpha}$ is a compatible topology for $B_{\alpha}$, it follows again that $\{x, y\}$ is not $\sigma$-connected. If there is no block of $G$ containing both $x$ and $y$, then there is some cut-point $z \notin\{x, y\}$ of $G$ such that $x$ and $y$ lie in different components of $G \backslash\{z\}$. That $\{x, y\}$ is discrete in $(G, \tau)$ now follows from (5) by considering the cases in which $z$ is maximal or minimal and whether or not one of the vertices $x$ or $y$ lies in the component of $G \backslash\{z\}$ which contains $v_{0}$.

Next we show that $\tau$ is a compatible topology for $G$. Since $\tau$ is weakly compatible, it follows from [6, Lemma 2.7] that it suffices to show that if $H$ is any $\tau$-connected subgraph of $G$ and $N(w) \cap H=\varnothing$, then $w \notin \mathrm{cl}_{\tau}(H)$. If $w \notin W$, then $w$ has a $\tau$-neighbourhood contained in $N(w)$ and the result follows trivially; hence we suppose that $w \in W$ and that $B_{\alpha}$ has been chosen as above, that is to say, $w$ is determining in the block $B_{\alpha}$ and if $w$ is special in some block, then $B_{\alpha}$ was chosen so that the distance of $v_{\alpha}$ to $v_{0}$ is minimal among all blocks in which $w$ is special. 
If $w \in \mathrm{cl}_{\tau}(H)$, then since $N(w) \cup o\left(v_{\alpha}\right)$ is a $\tau$-open neighbourhood of $w$ and $H \cap N(w)$ $=\varnothing$, it follows we can assume that $H \subseteq o\left(v_{\alpha}\right)$. There are two cases to consider.

(a) If $H$ meets some block $B$ containing $w$, then since $H \cap N(w)=\varnothing$ and $N(w)$ contains all maximal vertices of $B$, it follows that $H \cap B$ is a finite set of minimal vertices, say $z_{1}, \ldots, z_{n}$. By (5),o(zi) is $\tau$-closed for each $i \in\{1, \ldots, n\}$ and so for each such $z_{i}$, $H \cap o\left(z_{i}\right)=H \cap\left(o\left(z_{i}\right) \cup N\left(z_{i}\right)\right)$ is a $\tau$-clopen subset of $H$. Thus for some $i \in\{1, \ldots, n\}$, $H \subseteq o\left(z_{i}\right)$ and so $\left(N(w) \cup o\left(v_{\alpha}\right)\right) \backslash o\left(z_{i}\right)$ is a $\tau$-open neighbourhood of $w$ missing $H$.

(b) If on the other hand, $H \cap B=\varnothing$ for each block $B$ containing $w$, then for some block $B$ containing $w$ there is a vertex $z \in B$ such that $H \cap o(z) \neq \varnothing$, for otherwise, $H \cap$ $o\left(v_{\alpha}\right)=\varnothing$. Since $w$ is determining in each block which contains it, $z$ is not special in $B$. If $z$ is a minimal vertex, then since $H \cap o(z)=H \cap(o(z) \cup N(z))$, it follows from (5) that $H \cap o(z)$ is a $\tau$-clopen subset of $H$ and hence $H \subseteq o(z) \backslash\{z\}$. Thus $\left(N(w) \cup o\left(v_{\alpha}\right)\right) \backslash o(z)$ is a $\tau$-open neighbourhood of $w$ missing $H$. If on the other hand $z$ is maximal, then $H$ must meet some graph component $C \in \mathscr{C}_{z}$. However, again using (5), $C$ is $\tau$-closed and $C \cup\{z\}$ is $\tau$-open, thus since $H \cap C=H \cap(C \cup\{z\})$, it follows that $C \cap H$ is a proper $\tau$-clopen subset of $H$. Thus $H \subseteq C$ and then $G \backslash C$ is a $\tau$-open neighbourhood of $w$ which misses $H$.

Finally, we need to show that $(G, \tau)$ is compact. To this end, let $\mathscr{S}$ be a cover of $G$ by $\tau$-open sets. There is some $U_{0} \in \mathscr{S}$ such that $w_{0} \in U_{0}$. Since $w_{0}$ is a determining vertex for $B_{0}$ and $U_{0} \in \tau_{0}$, it follows that $U$ covers $G$ except for a finite number of sets $\left\{o\left(z_{i}\right): i \in\right.$ $\{1, \ldots, n\}\}$ for some finite set of minimal vertices $\left\{z_{i}: 1 \leq i \leq n\right\} \subseteq B_{0}$ and a finite number of graph components $\left\{C_{i}: 1 \leq y \leq m\right\}$ in $\cup\left\{\mathscr{C}_{z}: z \in K\right\}$ for some finite set $K$ of maximal vertices in $B_{0}$. Let $B_{\alpha_{i}}(1 \leq i \leq m)$ be the (unique) immediate successor block of $B_{0}$ which meets $C_{i}$. Since $z_{i}$ is determining in each block which contains it, $z_{i} \in W$ and each block $B_{\alpha_{i}}$ contains a determining vertex $w_{\alpha_{i}} \in W$. The above argument can now be repeated for the finite family of subgraphs $\left\{o\left(z_{i}\right): 1 \leq i \leq n\right\} \cup\left\{C_{i}: 1 \leq i \leq m\right\}$. Since the block-cutpoint tree of $G$ has no infinite paths, it follows from König's lemma (see [4, page 298]) that the process stops after a finite number of steps and we obtain a finite subcover of $\mathscr{Y}$, thus proving that $(G, \tau)$ is compact.

Conversely, suppose that $\tau$ is a compact compatible topology for $G$ and the block-cutpoint tree of $G$ has an infinite path. Then $G$ contains an infinite path of distinct vertices $P=\left\{p_{n}: n \in \mathbb{N}\right\}$. The intersection of this path with each block $B$ of $G$ must be finite since $B$ is either trivial or isomorphic to $K_{2, \gamma}$ for some cardinal $\gamma$. Hence, considering $P$ as an induced subgraph of $G$, each vertex $p_{n} \in P$ has only a finite number of graph neighbours and so $P$ is blunt. Thus by [5, Corollary 2.15], the only compatible topology for $P$ is the Alexandroff topology. Thus $\tau \mid P$ is the Alexandroff topology on $P$ and hence is locally finite; it follows that $(P, \tau \mid P)$ is not compact and hence is not closed in $(G, \tau)$.

However, $\mathrm{cl}_{\tau}(P)$ is compact, and if $y \in \mathrm{cl}_{\tau}(P) \backslash P$, then since the intersection of $P$ with each block is finite, it follows that $N(y) \cap P$ is finite. Thus $N(y)$ misses some final interval of $P$, say $N(y) \cap\left\{p_{n}: n \geq k\right\}=\varnothing$. Since $\tau$ is a compatible topology for $G$, it follows from [6, Lemma 2.7] that there is some $\tau$-neighbourhood $U$ of $y$ which misses the $\tau$ connected set $\left\{p_{n}: n \geq k\right\}$ and hence $U \cap P$ is finite. Thus each vertex of $\operatorname{cl}_{\tau}(P)$ has a $\tau$-neighbourhood whose intersection with $P$ is finite, contradicting the fact that $\mathrm{cl}_{\tau}(P)$ is compact. 
We note that in the final paragraph of the proof of Theorem 2.5, we have effectively shown that $G$ has an infinite path if and only if its block-cut-point tree has an infinite path and hence we have proved the following.

CoRollary 2.6. Let $(G,<)$ be a connected ordered bipartite graph in which all cycles are of length no greater than 4 . Then $(G,<)$ has a compact compatible topology if and only if $G$ has no infinite paths.

Since the operation of passing to the bipartite skeleton destroys all odd cycles, after applying Lemma 2.4 we have shown the following.

THEOREM 2.7. Let $(G,<)$ be an ordered comparability graph in which every cycle is of length at most 5, then $G$ has a compact compatible topology if and only if it has no infinite paths.

Since every minimal vertex of a finite block is determining, similar methods can be used to prove the following theorem, but we omit the laborious details.

Theorem 2.8. Let $(G,<)$ be an ordered comparability graph in which every block is finite, then $G$ has a compatible compact topology if and only if it has no infinite paths.

Example 2.9. For each $n \in \mathbb{N}$, let $A_{n}$ be a copy of $C_{6}$ (the 6-cycle) where we suppose that $V\left(A_{n}\right)=\left\{v_{1, n}, \ldots, v_{6, n}\right\}$. We denote the disjoint union of the graphs $A_{n}$ by $H$ and let $G$ be the quotient graph obtained from $H$ by identifying each of the vertices $\left\{v_{1, n}: n \in \mathbb{N}\right\}$ and each of the vertices $\left\{v_{4, n}: n \in \mathbb{N}\right\} . G$ is clearly 2-connected and since if $v \in V\left(A_{n}\right)$ either $v_{1, n} \notin N(v)$ or $v_{4, n} \notin N(v)$, it follows that $G$ is blunt. It is then a consequence of [5, Corollary 2.15], that the only compatible topology for $G$ is the Alexandroff topology. However, the Alexandroff topology (corresponding to either of the two possible orderings of $G$ ) is not compact since, as is easily seen, the set of minimal elements is infinite and discrete in the relative topology.

We have shown that there exists a bipartite graph with no infinite paths and all of whose cycles are of length 6 , but which has no compact compatible topology. Thus Theorem 2.5 and Corollary 2.6 do not generalize to comparability graphs, all of whose cycles are of length at most 6 so proving that Theorem 2.7 is the best possible result and together with Example 2.9 gives a complete answer to Problem 2.1.

An interesting question arises in regard to local connectivity of compact compatible topologies. The Alexandroff topology on a poset or ordered comparability graph is locally connected as is the topology $\tau$ constructed in Theorem 2.5. On the other hand, the trivial graph (no edges) on a countably infinite set of vertices has a compact compatible topology but no locally connected compact topology. However we do not know the answer to the following.

Problem 2.10. If a connected ordered comparability graph has a compact compatible topology does it have a locally connected compact compatible topology?

\section{Acknowledgments}

We thank the referee for his helpful comments. Research was supported by Consejo Nacional de Ciencia y Tecnología México, Grant 38164-E. The second author wishes to 
thank the University of Auckland for their hospitality during the preparation of this paper. Victor Neumann-Lara died on February 26, 2004.

\section{References}

[1] C. Berge, Graphs and Hypergraphs, North-Holland Mathematical Library, vol. 6, NorthHolland, Amsterdam, 1973.

[2] R. Engelking, General Topology, Sigma Series in Pure Mathematics, vol. 6, Heldermann, Berlin, 1989.

[3] F. Harary, Graph Theory, Addison-Wesley, Massachusetts, 1957.

[4] A. Lévy, Basic Set Theory, Springer, Berlin, 1979.

[5] V. Neumann-Lara and R. G. Wilson, Compatible connectedness in graphs and topological spaces, Order 12 (1995), no. 1, 77-90.

[6] Compact compatible topologies for posets and graphs, Order 15 (1998), no. 1, 35-50.

[7] P. Préa, Graphs and topologies on discrete sets, Discrete Math. 103 (1992), no. 2, 189-197.

Victor Neumann-Lara: Instituto de Matemáticas, Universidad Nacional Autónoma de México, Ciudad Universitaria, 04510 México DF, Mexico

Richard G. Wilson: Departamento de Matemáticas, Universidad Autónoma Metropolitana, Unidad Iztapalapa, Avenida San Rafael Atlixco 186, Apartado Postal 55-532, 09340 México DF, Mexico

E-mail address:rgw@xanum.uam.mx 


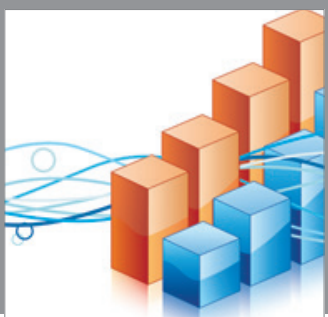

Advances in

Operations Research

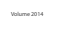

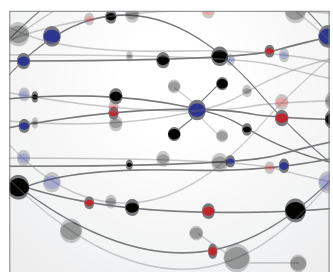

\section{The Scientific} World Journal
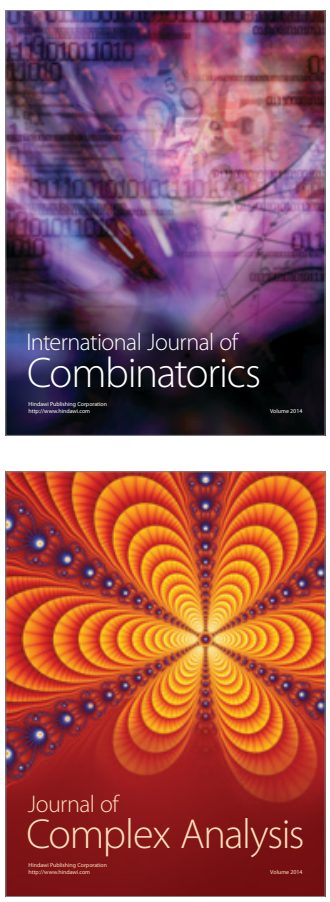

International Journal of

Mathematics and

Mathematical

Sciences
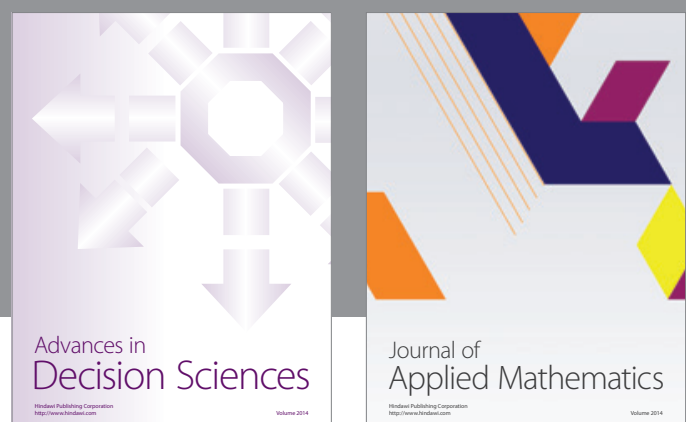

Journal of

Applied Mathematics
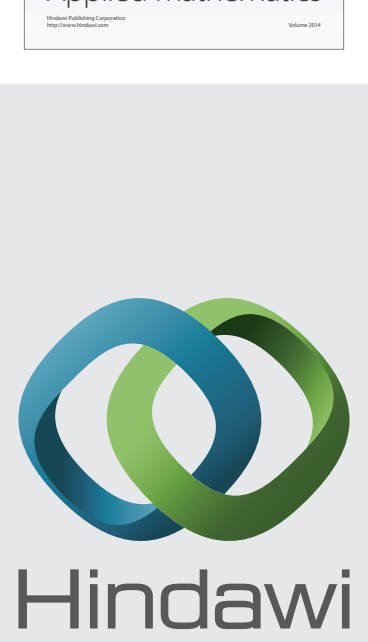

Submit your manuscripts at http://www.hindawi.com
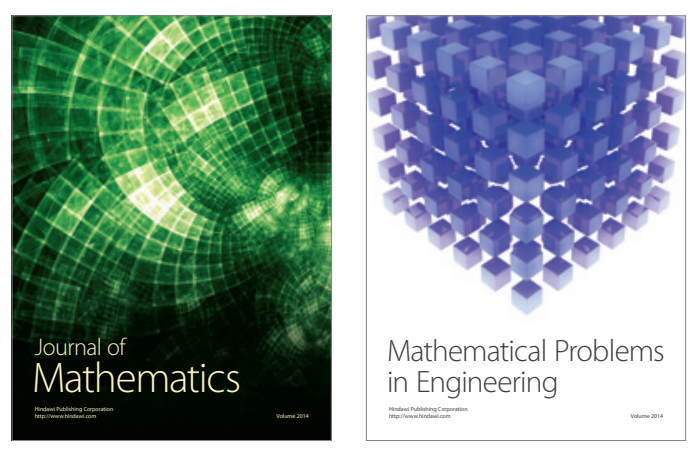

Mathematical Problems in Engineering
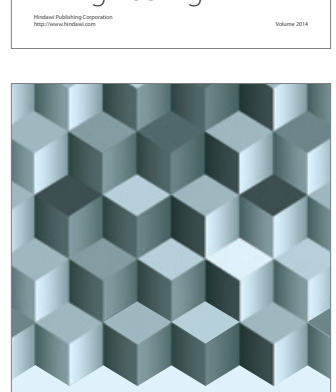

Journal of

Function Spaces
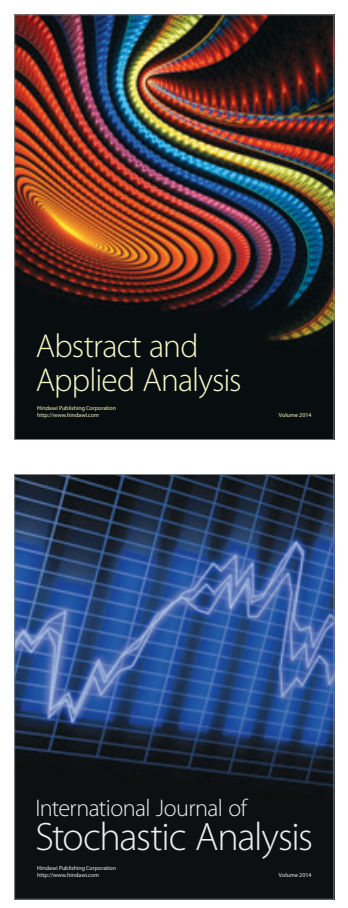

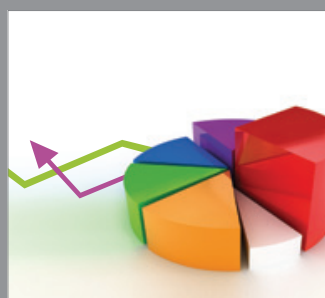

ournal of

Probability and Statistics

Promensencen
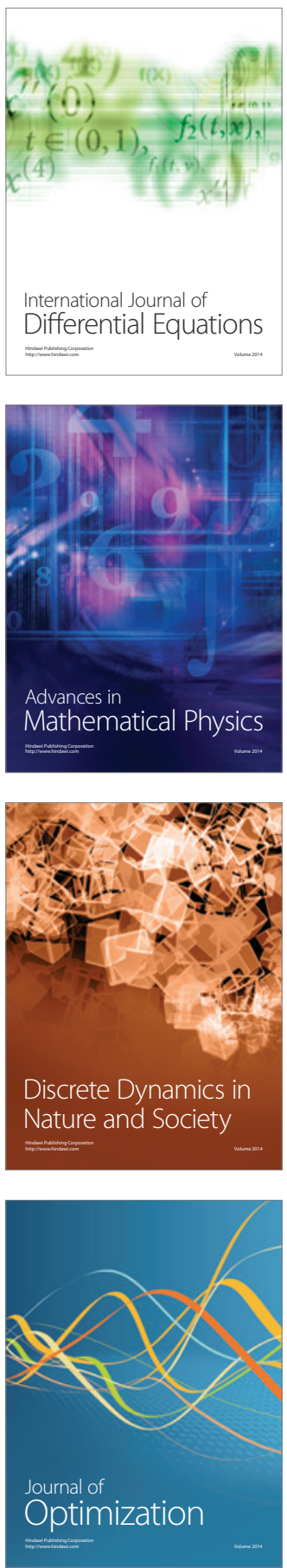